\title{
Diseño y valoración de una audioguía multilingüe y accesible
}

\author{
José Tomás Conde Ruano
}

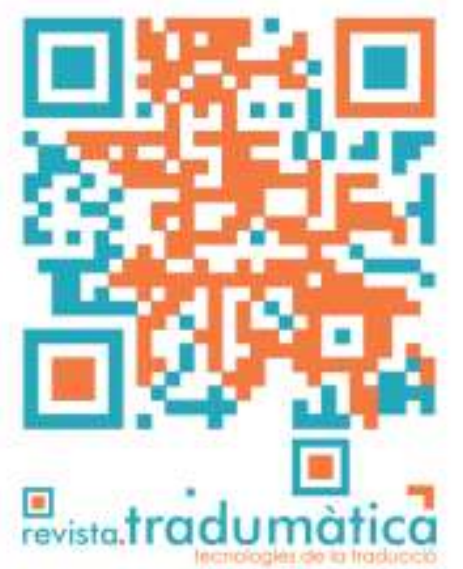

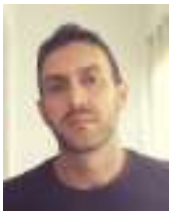

José Tomás Conde Ruano

Universidad del País Vasco (UPV/EHU)

tomas.conde@ehu.eus; ORCID:

0000-0002-4089-5019

\section{Resumen}

Este trabajo describe y valora el producto desarrollado en virtud de un proyecto de innovación educativa cuyo objetivo es la creación de una audioguía multilingüe, accesible e inclusiva, de la Facultad de Letras de la Universidad del País Vasco (UPV/EHU). Basada en las metodologías de aprendizaje-servicio, la audioguía es una iniciativa conjunta de los departamentos de Historia del Arte y Filología Inglesa y Alemana y Traducción e Interpretación, con la que se pretende asistir a las personas con discapacidad visual para que se muevan con seguridad a lo largo de los espacios arquitectónicos que conforman el edificio.

Tras la introducción, se aborda la audiodescripción y su relación con el concepto de accesibilidad, el papel de las nuevas tecnologías en esta modalidad y las iniciativas que se han puesto en marcha sobre este tema desde los entornos formativos. A continuación, el trabajo se centra en los aspectos técnicos de la audioguía.

En primer lugar, se relatan los pasos seguidos para configurar y alojar la herramienta en una página web institucional. En segundo lugar, se muestra la situación actual de dicha página web: su aspecto, el diseño elegido y la experiencia de navegación. En tercer lugar, se pretende realizar una autoevaluación del producto a partir del modelo propuesto por Conde (en prensa). El objetivo es comprobar si la audioguía a) encaja con la normativa existente al respecto y con lo que los expertos esperan de una herramienta semejante; b) funciona respecto a los objetivos con que fue creada, a partir de la experiencia de destinatarios potenciales de la misma y c) compensa a los agentes que han propiciado su creación y, en especial, al equipo del proyecto.

La tecnología resulta clave para la formación de traductores e intérpretes, así como para su desempeño profesional. Los beneficios son todavía más evidentes cuando la tecnología se pone a disposición de iniciativas para mejorar la accesibilidad. Más allá de ilustrar el resultado de una experiencia concreta y de ofrecer un marco para la evaluación de la calidad de este tipo de productos, el presente trabajo podría extrapolarse a otros campos y espacios e incluso inspirar la creación de herramientas y metodologías específicas para traducir los textos audiodescritos. 
Palabras clave: accesibilidad; AD; audioguía; proyecto de innovación educiativa; interacción persona-ordenador; calidad; evaluación

Resum

Aquest treball descriu i valora el producte desenvolupat en virtut d'un projecte d'innovació educativa, l'objectiu del qual és la creació d'una audioguia multilingüe, accessible i inclusiva, de la Facultat de Lletres de la Universitat del País Basc (UPV/EHU). Basada en les metodologies d'aprenentatge-servei, l'audioguia és una iniciativa conjunta dels departaments d'Història de l'Art, de Filologia Anglesa i Alemanya i de Traducció i Interpretació, amb la qual es pretén assistir les persones amb discapacitat visual perquè es moguin amb seguretat pels espais arquitectònics que conformen l'edifici.

Després de la introducció, abordem l'audiodescripció i la relació amb el concepte d'accessibilitat, el paper de les noves tecnologies en aquesta modalitat $\mathrm{i}$ les iniciatives que s'han posat en marxa sobre aquest tema des dels entorns formatius.

A continuació, el treball se centra en els aspectes tècnics de l'audioguia. D'entrada, es relaten els passos seguits per configurar i allotjar l'eina en un lloc web institucional. En segon lloc, es mostra la situació actual del web: l'aspecte, el disseny escollit i l'experiència de navegació. En tercer lloc, es pretén realitzar una autoavaluació del producte a partir del model proposat per Conde (en premsa). L'objectiu és comprovar si l'audioguia a) encaixa amb la normativa existent i amb allò que els experts esperen d'una eina d'aquestes característiques; b) funciona quant als objectius amb què ha estat creada, a partir de l'experiència de destinataris potencials i c) compensa els agents que n'han propiciat la creació i, especialment, l'equip del projecte.

La tecnologia resulta clau per a la formació de traductors i intèrprets, així com per a l'exercici de la seva professió. Els beneficis són encara més evidents quan la tecnologia es posa a l'abast d'iniciatives per millorar l'accessibilitat. Més enllà d'illustrar el resultat d'una experiència concreta i d'oferir un marc per a l'avaluació de la qualitat d'aquest tipus de productes, aquest treball podria extrapolar-se a altres camps i espais i, fins i tot, inspirar la creació d'eines i metodologies específiques per traduir els textos audiodescrits.

Paraules clau: accessibilitat; AD; audioguia; projecte d’innovació educativa; interacció personaordinador; qualitat; avaluació

Abstract

This article describes and assesses a product that was developed as part of an educational innovation project aimed at creating an accessible and inclusive multilingual audio guide for the Faculty of Arts at the University of the Basque Country (UPV/EHU). This audio guide stems from a service learning approach and is a joint initiative by the 
faculty's departments of History of Art, English and German Philology and Translation and Interpreting. The objective is to provide support for visually impaired people so that they can move safely through the various architectural spaces in the faculty building.

The introduction is followed by a discussion on audio description and how it relates to the notion of accessibility, the role of new technologies in this modality and the schemes that have been put into operation in this area in educational environments.

The article then goes on to focus on the technical aspects of the audio guide: first, a description of the steps to configure and install the tool on an institutional website; second, the current version of the web page is shown (appearance, the design chosen and the browsing experience); and third, a self-assessment of the product based on the model proposed by Conde (in press). The aim is to check whether the audio guide a) complies with existing regulations and what experts expect from such a tool; b) works regarding the objectives for which it was created based on the experience of potential end users; and c) the reward for the agents that have promoted its creation and, in particular, the project team.

Technology is key to training translators and interpreters, as well as to how they perform professionally. The benefits are even more evident when technology is placed at the disposal of initiatives to improve accessibility. In addition to illustrating the results of a specific project experience and offering a framework for assessing the quality of this kind of product, this study could be extrapolated to other fields and spaces and even inspire the creation of specific tools and methodologies for translating audio-described texts.

Keywords: accessibility; audiodescription; audioguide; teaching innovation project; human-computer interaction; quality; evaluation

\section{Introducción}

La accesibilidad y la audiodescripción (AD) están cada vez más presentes en la sociedad, gracias al empuje de organismos internacionales - por ejemplo, Naciones Unidas (Remael et al., 2019) - y gobiernos nacionales, como el de España (Carlucci y Seibel, 2014) o el de Perú (Castro Fernández et al., 2018), entre otros.

Los Estudios de Traducción (EdT) no son ajenos a esta tendencia, como muestran, por ejemplo, el espacio dedicado en números especiales de revistas como MonTI (núm. 12, de 2020), TRANS (núm. 24, de 2020) y Linguistica Antverpiensia (núm. 18, de 2019) y la proliferación de títulos de posgrado en los que se abordan estos conceptos, como los recogidos por Sanz-Moreno (2020).

A pesar de este interés, apenas se han audiodescrito espacios arquitectónicos completos de los propios edificios universitarios, a excepción de dos facultades de la Universidad de Granada (véase, por ejemplo, Soler Gallego y Luque Colmenero, 2016) y 
la experiencia monolingüe dirigida por Juan José Martínez Sierra en la Universitat de València. Frente a esta última, la audioguía presentada en este trabajo tiene la particularidad de que no solo es accesible, sino que también es multilingüe. Frente a la primera, la audioguía creada para la UPV/EHU emplea la representación de tipo mapa para casi todo el edificio; es decir: no se crean rutas prefijadas (como en la de Granada), sino que las crea el propio usuario en función de sus necesidades o intereses. Además, en el caso que se presenta, se parte de los estudios sobre calidad en traducción para proponer un protocolo concebido para valorar o testar esta y otras audioguías.

La sección 2 define los conceptos de accesibilidad y AD, así como la relación de estos con la tecnología, la formación y la innovación. Después, se describe el proceso de creación y el estado actual en que se encuentra la audioguía (§ 3), así como el sistema de testeo ( $(4)$. Las últimas dos secciones incluyen las conclusiones ( $§ 5$ ) y la bibliografía (§ 6).

\section{Marco teórico}

El objetivo de esta sección es dibujar el entorno que inspira la creación de la audioguía que será descrita y testada después. Partiendo de una definición de los conceptos de accesibilidad y $A D$, se aborda el papel que juegan las herramientas tecnológicas (especialmente las audioguías) en estas prácticas, así como la presencia de estos conceptos en la formación de traductores e intérpretes, plasmándose sobre todo en la forma de proyectos de innovación educativa.

\subsection{Accesibilidad y $A D$}

La 'accesibilidad', que ha ido ampliando su alcance para incluir no solo a personas con discapacidad, sino también a aquellas con capacidades cognitivas y físicas diversas (López-Rodríguez et al., 2009), es definida por Zhang (2019: 74) como:

[...] a precondition intended to ensure that persons with a disability enjoy full and equal participation in society. Accessibility is the 'ability to access'. It primarily refers to designs for people with disabilities [...]

La accesibilidad se aplica en distintos ámbitos: desde la navegación internáutica (López-Rodríguez et al., 2009) hasta las artes escénicas (Remael et al., 2019), pasando por la propia traducción, donde se considera «una estrategia y una herramienta para crear textos que permitan el acceso a la información»(Carlucci y Seibel, 2014: 52).

Por otra parte, la $A D$ es un tipo de traducción intersemiótica (Castro Fernández et al., 2018) que consiste en la conversión de imágenes en palabras, de manera que las personas con déficit de visión interpreten la información no verbal de la manera más semejante posible a como lo hacen quienes ven correctamente (AENOR, 2005; ONCE, 2003).

Teniendo en cuenta la relevancia social de estos conceptos, no es de extrañar que en la universidad haya crecido el interés por su estudio. Tanto Mazur y Vercauteren (2019) como Zhang (2019) recogen proyectos en esta línea: por ejemplo, ACT (Matamala 
y Orero, 2016), orientado a la accesibilidad en la cultura, y ADLAB PRO (2017), sobre la creación de materiales didácticos para los audiodescriptores.

\subsection{Accesibilidad y tecnología}

La mayoría de los proyectos de investigación en la universidad se apoyan en el uso de las nuevas tecnologías para alcanzar la accesibilidad. Lo que las personas con discapacidad no logran fácilmente por sí mismas lo consiguen de un modo más eficaz gracias a la interacción persona-ordenador.

Se ha analizado dicha interacción sobre todo en dos direcciones: los factores humanos -o cómo interactúan las personas con las herramientas- y la ergonomía. En palabras de O’Brien (2012: 103-104):

Human factors focuses on how people interact with tools and technology. While the term 'ergonomics' traditionally referred to the ease with which hardware, such as keyboards, could be used, it seems to have evolved to also include the 'ease' with which software products can be used.

Prieto Velasco (2009) subraya el papel de la tecnología para transmitir información tanto al receptor prototípico como a públicos dispares, mientras que Agulló et al. (2018) consideran que los dos ejes fundamentales para el avance de la accesibilidad son la tecnología y los usuarios finales.

La interacción persona-ordenador estimula el avance de los procesos sociales; como comentan Risku y Windhager (2013: 36):

[...] it is not only the interaction of human actors that produces change but also the interaction of human actors and nonhuman actants. In translation today, the influence of non-human actants on the translation process and product, in other words, on what is translation, cannot be underestimated.

Remael et al. (2019) citan ejemplos en los que los avances tecnológicos permiten el acceso a las artes, a saber: vídeos introductorios en lenguaje de signos, auriculares de uso individual, tabletas colocadas en los asientos del teatro, teléfonos inteligentes, aplicaciones de móvil y tecnologías inmersivas. Siguiendo con las artes escénicas, Hermosa-Ramírez, que señala la creciente importancia de los estudios de AD basados en usuarios (2020), considera una forma de interacción persona-ordenador la conjunción de la traducción y la $A D$ parcialmente en directo en obras teatrales.

No obstante, la audioguía es la herramienta más común para facilitar la accesibilidad de las personas ciegas, ya en los espacios y representaciones físicas, ya en los medios audiovisuales. Además, su uso en móviles está especialmente recomendado, dadas las posibilidades que este medio ofrece para las personas con discapacidad sensorial (Soler Gallego y Chica Núñez, 2014).

\subsection{Audioguías e innovación docente}

Dado el auge de los contenidos multimodales, la información con la que trabajan los traductores integra más aspectos visuales. Los programas accesibles son también cada vez más numerosos, por lo que se hace necesaria la formación en accesibilidad (Prieto Velasco, 2009; Sanz-Moreno, 2020). En los Estudios de Traducción, las iniciativas 
formativas pueden articularse de muchas maneras; en el caso descrito por Luque Colmenero y Soler Gallego (2019), por ejemplo, se proponen prácticas colaborativas en museos.

Habituales son asimismo las experiencias generadas a partir de proyectos de innovación docente. La guía detallada por el propio Prieto Velasco (2009) es fruto de uno de estos proyectos. Otros ejemplos son las metodologías de trabajo colaborativo descritas por Cerezo Merchán y De Higes Andino (2013); la elaboración de una guía multimedia accesible, en Carlucci y Seibel (2014), y el proyecto complejo de AD de elementos artísticos y arquitectónicos, en Carlucci y Seibel (2016).

El presente trabajo culmina un proyecto de innovación educativa (PIE) puesto en marcha en la UPV/EHU con el objetivo de crear una audioguía accesible, inclusiva y multilingüe de su Facultad de Letras. Como en la experiencia relatada en Mendoza y Matamala (2019), las etapas previas incluyen, entre otras, la redacción y revisión del guion y su traducción, locución y montaje.

Una vez terminada la versión 1.0 de la audioguía, es pertinente valorarla, tanto como proceso como en calidad de producto. Una mirada crítica puede ayudar a tomar decisiones que la mejoren e, incluso, sirvan de inspiración para otras audioguías que puedan diseñarse en el seno de la UPV/EHU y aun en otras instituciones. Antes de la valoración, se describe brevemente el estado actual del producto y los pasos que se han seguido para crearlo.

\section{La audioguía}

Basada en las metodologías de aprendizaje-servicio (Tapia, 2010), la audioguía de la Facultad de Letras (alojada aquí: http://www.ehu.eus/letrapk/apk/audiogida/es/) es una iniciativa conjunta de los departamentos de Historia del Arte y Filología Inglesa y Alemana y Traducción e Interpretación. Con ella, se asiste a las personas con discapacidad visual para que se muevan con seguridad a lo largo de los espacios arquitectónicos que conforman el edificio.

Esta sección se ocupa, en primer lugar, de las acciones tomadas con objeto de alojar la audioguía en una página web de la UPV/EHU; a continuación, se detalla su aspecto actual; y, para terminar, se comenta la experiencia de navegación.

\subsection{Creación}

La página web se creó al mismo tiempo que se grababan los audios de la planta baja. Para estas locuciones se utilizó el laboratorio de interpretación de la facultad, que dispone del sistema Televic, cabinas y ordenadores con el programa Audacity. Era la opción más lógica, habida cuenta de que los locutores, que eran alumnos de las asignaturas de interpretación, estaban más que familiarizados con dicha infraestructura y que el sistema garantizaba unas grabaciones de buena calidad. No obstante, después de esta primera tanda llegaron la pandemia y el consiguiente confinamiento, lo que 
provocó que algunas de las demás grabaciones tuvieran que realizarse a distancia, desde el ordenador de los locutores.

Se puede hablar de tres fases en la creación de la versión 1.0 de la audioguía. En la primera, se monta la página y se suben los audios de la planta baja; en la segunda, se van incorporando los demás audios de manera paulatina; y, en la tercera, se corrigen errores de la apariencia y del contenido (véase § 4.1).

La creación de la página web corrió a cargo de un técnico (en adelante, TP) de la facultad, miembro también del PIE. Para la programación, utilizó un editor de texto combinando HTML, CSS y JavaScript. Estos lenguajes se eligieron porque permiten el acceso a la audioguía desde el navegador de cualquier dispositivo móvil.

\subsection{Diseño}

En cuanto al diseño, se prescindió de logotipos e imágenes para que las personas ciegas pudieran acceder a todos los contenidos. La interfaz muestra poco texto, de modo que los usuarios pueden localizar rápido el contenido que más les interesa. Se buscó el mayor contraste posible, utilizando un fondo negro con letras blancas que impide que los usuarios con resto de visión se deslumbren al leer.

Para que los usuarios pudieran controlar la velocidad de lectura, se valoró incluir las descripciones en lugar de las locuciones y utilizar el lector de textos para escucharlas. Sin embargo, al final se apostó por los locutores humanos, primándose el valor pedagógico de la experiencia para los estudiantes de interpretación que locutarían los textos.

La estructura de la audioguía es simple para que se pueda navegar por ella sin obstáculos, de modo que, por ejemplo, se descartó la idea de separar las plantas del edificio en páginas distintas. Así pues, cuenta solo con tres secciones, una por cada idioma (español, inglés y vasco). Cada sección tiene dos páginas: en la primera están las locuciones, divididas en apartados (según las plantas del edificio); en la segunda, los créditos de los participantes en el proyecto.

\subsection{Navegación}

El enlace en la página de la facultad se realiza desde la versión de la web a su idioma correspondiente; no obstante, es posible cambiar de idioma desde la propia audioguía. Otra opción personalizable es la visualización: al estar diseñado con CSS adaptativo, se puede ajustar a las dimensiones de distintos soportes.

La audioguía tiene ciertas limitaciones técnicas. La principal es que requiere de conexión a Internet. Una vez dentro de la web, el usuario ciego no puede controlar el audio de las locuciones; tampoco puede volver a un punto concreto sin escuchar la locución desde el principio, ni avanzar rápidamente por la pista. El usuario con resto de visión sí dispone de una barra de progreso que le muestra la duración del corte seleccionado y que le permite, asimismo, avanzar o retroceder. Otra ventaja es que los 
usuarios no necesitan descargar los archivos si solo están interesados en escuchar la locución de un espacio concreto.

Para terminar, una posibilidad que no se descarta para el futuro $-\mathrm{y}$ que se barajó en un primer momento- es convertir la audioguía en una aplicación para móviles. En cualquier caso, la versión actual funciona como si fuera una aplicación; de hecho, la página web accesible desde el móvil es también la opción que se está planteando para otros proyectos, como el de Castro Fernández et al. (2018), sobre museos.

Si bien la audioguía está completada, siempre es aconsejable testarla para identificar mejoras, que se podrían aplicar en configuraciones o iniciativas posteriores. Para ello, es preciso realizar pruebas con aquellas personas que pueden tener interés en su uso, en especial con personas ciegas.

\section{Valoración}

En esta sección se exponen las pruebas que se han efectuado sobre el proceso y el producto completados. Ninguno de los recursos bibliográficos afines aborda específicamente las audioguías de espacios arquitectónicos. Además, en los recursos existentes (como los citados en el párrafo siguiente) se mezclan directrices relativas al proceso y al producto y no se distingue entre las comprobaciones que deben ejecutar los diseñadores de los productos, el personal técnico y los usuarios potenciales. Por todo ello, se crearon herramientas ad hoc adaptadas al proyecto, a partir de otras guías, estándares y recomendaciones sugeridas por expertos en accesibilidad.

Se ha partido de la norma UNE 153020, que establece los requisitos para un producto audiodescrito de calidad (Cerezo Merchán y De Higes Andino, 2013). No obstante, dada su antigüedad y falta de integridad, se ha recurrido también a guías y trabajos orientados a museos (CESyA, 2008), teatro (CESyA, 2013; Hermosa-Ramírez, 2020), televisión (Álvarez Álvarez, 2014) y páginas web (López-Rodríguez et al., 2009 o Prieto Velasco, 2009). Entre los criterios incluidos en las herramientas puede hallarse también la influencia de los cuatro elementos que Snyder (2008) destaca para el desarrollo de todo tipo de AD: observación atenta, selección de información, lenguaje eficaz y habilidades orales.

La evaluación se ha estructurado a partir del modelo propuesto por Conde (en prensa). Primero, se ha cotejado el producto con las normas existentes; después, se ha probado su funcionalidad; $y$, por último, se ha analizado el proceso en relación con su rentabilidad.

\subsection{Eje normativo}

La primera comprobación midió el eje normativo; esto es: cómo encajan el producto y el proceso en el contexto normativo en el que han surgido. Castro Fernández et al. (2018) exponen una idea similar para evaluar la AD de textos museísticos. Para la presente valoración, se seleccionaron las recomendaciones que cabía testar sin la ayuda del público objetivo. Respecto al formato, se optó por una lista de comprobación. Al tratarse de un testeo técnico de la web, se tuvieron en cuenta los cuatro principios 
recogidos por Prieto Velasco (2009): perceptibilidad, operatividad, inteligibilidad y coherencia.

La comprobación normativa se completó en varias fases, ya que algunas dudas requirieron la consulta posterior con el TP. La Tabla 1 muestra la lista de comprobación, donde $\mathrm{CO}$ se refiere a la opinión del coordinador del proyecto; TP, a la opinión del técnico y $\mathrm{Fl}$, a la respuesta final.

\begin{tabular}{|c|c|c|c|c|c|}
\hline Principios & Cód. & Criterios & $\mathrm{CO}$ & TP & $\mathrm{FI}$ \\
\hline \multirow{2}{*}{ Perceptibilidad } & $1 a$ & $\begin{array}{l}\text { Equivalencia textual para todos los elementos } \\
\text { no textuales }\end{array}$ & $\checkmark$ & $\checkmark$ & $\checkmark$ \\
\hline & $1 b$ & Dirección de contacto & $x$ & $x$ & $\checkmark$ \\
\hline \multirow{3}{*}{ Operatividad } & $1 c$ & $\begin{array}{l}\text { Disponible cuando surgen objetos } \\
\text { problemáticos }\end{array}$ & ? & $\checkmark$ & $\checkmark$ \\
\hline & $1 d$ & Se evita el movimiento & 8 & $\checkmark$ & $\checkmark$ \\
\hline & $1 e$ & Se permite parar en cualquier momento & $\checkmark$ & $\checkmark$ & $\checkmark$ \\
\hline \multirow{9}{*}{ Inteligibilidad } & If & Contraste de colores & 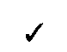 & $\checkmark$ & $\checkmark$ \\
\hline & $1 g$ & Fuentes claras & $?$ & $\checkmark$ & $\checkmark$ \\
\hline & $1 \mathrm{~h}$ & Máximo 2 tamaños de fuente & $\checkmark$ & $\checkmark$ & $\checkmark$ \\
\hline & $1 i$ & Tamaño grande o configurable & $\checkmark$ & $\checkmark$ & $\checkmark$ \\
\hline & $1 j$ & Dividido en bloques pequeños de información & $\checkmark$ & $\checkmark$ & $\checkmark$ \\
\hline & $1 k$ & Sin mayúsculas ni cursiva & $x$ & $x$ & $?$ \\
\hline & 11 & Sin justificar a la derecha & $\checkmark$ & $\checkmark$ & $\checkmark$ \\
\hline & $1 m$ & Sin demasiada información en cada página & $?$ & $\checkmark$ & $?$ \\
\hline & $1 n$ & Sin partir la información entre páginas & $\checkmark$ & $\checkmark$ & $\checkmark$ \\
\hline Coherencia & $1 \tilde{n}$ & Con pautas de repetición & $x$ & $x$ & $\checkmark$ \\
\hline
\end{tabular}

Tabla 1. Lista de comprobación técnica

Durante la primera fase -acometida por el propio $\mathrm{CO}$ - se constató que la mayoría de los criterios de calidad se cumplían. No obstante, se consultaron con el TP que había diseñado la página algunas cuestiones sobre las que surgieron dudas. 
Respecto al criterio 1c, TP replicó que depende del problema que surja; así, la audioguía no funciona si falla el servidor, pero, si desaparecen los estilos de la web, la página sigue siendo funcional. Sobre la fuente $(1 g)$, TP confirmó que, por su seguridad, la escogida fue Arial y que, si el usuario no cuenta con ella, su dispositivo recurre a otro tipo de letra paloseco, que agiliza la lectura en pantalla. En cuanto a 1n, CO dudó de si no habría sido mejor colocar la información de cada planta en una página diferente. TP recordó que se había hecho así para facilitar una visión completa de la audioguía. Ambos coincidieron en que sería apropiado preguntar a los usuarios finales qué opción les parecía mejor.

La lista de comprobación ayudó a que TP corrigiera también algunas cuestiones. Por ejemplo, se vio que no aparecía ninguna dirección de contacto (1b). Para CO, no había coherencia en la disposición de los botones (1ñ) que, dentro de cada planta, se referían a las alas derecha e izquierda del edificio. Tras consultarlo con TP - que, en cambio, no creía que la disposición incoherente fuera un problema importante para el público objetivo-, se confirmó y subsanó este punto. Por último, la audioguía contravenía lo leído en la bibliografía respecto al criterio $1 k$. TP argumentó que, al tratarse de segmentos hiperbreves, se utilizó la mayúscula, ampliando el interletraje. En cualquier caso, se decidió preguntar sobre este punto también a los usuarios potenciales, en la siguiente comprobación.

\subsection{Eje funcional}

La segunda comprobación midió el eje funcional; esto es: cómo funciona la audioguía respecto a los objetivos para los que fue creada. Díez Carbajo (2018) aboga por evaluar la usabilidad basándose primero en la observación del comportamiento de los usuarios mientras interactúan con las máquinas y, en segundo lugar, en encuestas o entrevistas. Esta sección sigue el mismo orden: se explica el recorrido seguido por los participantes y, a continuación, se detalla la información obtenida con los cuestionarios que completaron tras su experiencia por diversos espacios de la facultad.

\subsubsection{Recorrido}

Los usuarios son los beneficiarios de la accesibilidad, con lo cual su participación resulta altamente recomendable (Luque Colmenero y Soler Gallego, 2019). También en el trabajo de Remael et al. (2019) intervienen personas ciegas en la valoración de su curso MOOC. Agulló et al. (2018) justifican en la misma Convención Internacional sobre los Derechos de las Personas con Discapacidad la participación de estas personas en los procesos de testeo.

Dado el contexto pandemiológico, se temía que fuera imposible contar con usuarios potenciales distintos de aquellos que habían estado presentes en fases anteriores del PIE. En primer lugar, con la ayuda del Servicio de Atención a Personas con Discapacidades de la UPV/EHU, se envió un mensaje a los ocho alumnos con discapacidad visual del Campus de Álava, al cual respondieron dos. En segundo lugar, apareció una voluntaria que conoció el proyecto por medio de una de las alumnas ciegas que habían participado en las primeras fases del PIE. En tercer lugar, esta 
voluntaria, una vez vivida la experiencia, se la contó a su pareja, que también se animó a participar. En total, cuatro usuarios potenciales probaron la audioguía.

La prueba se desarrolló en la facultad en diciembre de 2020. Tres informantes acudieron por la tarde del día 10 y el otro, a la tarde siguiente. Con las dos estudiantes, CO se citó en la puerta de la fachada principal. Con los otros dos sujetos, CO se acercó a un lugar céntrico de la ciudad para recogerlos y acompañarlos hasta la entrada de la facultad. En cualquier caso, la prueba fue similar para los cuatro: un recorrido informal, acompañados solamente por $\mathrm{CO}$, y accediendo a la audioguía a través de un teléfono móvil con acceso a Internet. Para guiar el paseo, se propusieron tres hitos parciales. Los usuarios debían:

1. en la planta baja, llegar hasta la conserjería (donde, por motivos de rastreo, los dos sujetos ajenos a la UPV/EHU estaban obligados a registrarse);

2. desde la escalera derecha del segundo piso, llegar hasta un aseo;

3. desde la escalera izquierda del primer piso, terminar el paseo en el despacho de CO.

De un hito al siguiente, los sujetos fueron llevados del brazo por CO, directamente y sin atender a la AD: con la prueba no se pretendía registrar al detalle cada movimiento de los usuarios, sino más bien ofrecer una oportunidad para que estos se hicieran una idea de las características de la audioguía, así como de sus posibilidades.

Los usuarios tardaron entre 35 y 45 minutos en completar el recorrido. Mientras lo hacían, destacaban aspectos arquitectónicos de la facultad ajenos al proyecto, como la inclusión del braille en la señalización de despachos y aseos, la amplitud de los pasillos principales o la distribución lógica de los espacios (las alas izquierda y derecha son prácticamente simétricas). Al terminar, se les pidió que, ya en casa, navegaran por la página de la audioguía, para que escucharan más grabaciones y, si lo deseaban, reprodujeran las del resto de idiomas (los cuatro eligieron la versión en español para el paseo). Recibirían un enlace a un cuestionario electrónico cuya accesibilidad había sido testada por otra exalumna con discapacidad visual.

\subsubsection{Cuestionario}

Para incluir al público objetivo en los procesos de valoración pueden darse diversas opciones. En la investigación de Álvarez de Morales (2017), las grabaciones realizadas fueron valoradas por una persona con discapacidad visual, cuya opinión se recogió después mediante un cuestionario y una entrevista. Se utilizó el primero para recabar datos sociodemográficos y de hábitos previos con herramientas accesibles, mientras que la entrevista resultó eficaz para conocer aspectos relacionados con la información presente en las grabaciones.

El cuestionario o encuesta es un método útil, pues ofrece datos de una naturaleza altamente estructurada. Lo han utilizado García-Prieto (2020) y Zhang (2019) para recoger datos de los usuarios, aunque, en este último caso, se complementó con grupos focales. Cacheiro Quintas (2019) utiliza una encuesta para medir la característica que más le interesa acerca de la calidad: la usabilidad, que considera clave para la interacción persona-ordenador. Coincide con Díez Carbajo (2018) en que la mediación entre el ser 
humano y la máquina se produce a través de la interfaz, mediante la cual los usuarios logran realizar tareas, reducir sus limitaciones y aumentar su motivación.

El trabajo de Cacheiro Quintas cita una serie de criterios de calidad para valorar una plataforma formativa de subtitulado: inteligibilidad, aprendizaje, operabilidad, protección frente a errores, estética y accesibilidad. Además, distingue entre pruebas de usabilidad objetivas y subjetivas. Las evaluaciones objetivas analizan datos sin una interacción directa de los usuarios; en cambio, las subjetivas consideran la usabilidad percibida por los participantes a partir de entrevistas o cuestionarios. En su estudio realiza evaluaciones de los dos tipos. Los datos objetivos corresponden al tiempo que lleva superar cada escenario y al porcentaje de éxito; los subjetivos se recabaron mediante una herramienta adaptada del cuestionario QUIS de la Universidad de Maryland (Cacheiro Quintas, 2019), elegido por su conveniencia para el estudio: era fiable, universal y adaptable al formato electrónico.

El cuestionario adaptado de Cacheiro Quintas sirvió de inspiración para el del presente estudio. Se aprovechó la bibliografía reseñada (CESyA, 2013, 2008; AENOR, 2005; Mendoza y Matamala, 2019; López-Rodríguez et al., 2009; Prieto Velasco, 2009) para añadir a las preguntas genéricas otras específicas sobre cuestiones como: estructura de frases, claridad de estilo, calidad de sonido, habilidades vocales, tono y registro, montaje y audionavegación, disponibilidad de recursos, inclusión de fases o elementos, profundidad de descripción, acceso, interactividad y operabilidad.

Tras la introducción y el consentimiento, la primera sección del cuestionario recaba cuatro datos sociológicos. Todos los sujetos afirmaron tener experiencia previa con audioguías y con otro tipo de aplicaciones tecnológicas de accesibilidad. Por lo demás, estos eran sus perfiles:

A. Agente comercial de la ONCE, de 19 años. Ceguera total.

B. Estudiante de Trabajo Social, de 44 años. Con rastro de visión, podía desplazarse por sí misma.

C. Estudiante de Filología Inglesa, de 18 años. Ceguera total. Única informante que había tenido algún contacto con la Facultad de Letras.

D. Periodista, de 27 años. Ceguera total.

La matriculada en la Facultad de Letras (C) no estaba demasiado familiarizada con el edificio, ya que estaba en primer curso y, debido a las restricciones impuestas por el Covid-19, apenas si había asistido de manera presencial. Del resto, solo B conocía el campus y D ni siquiera vivía en la misma ciudad.

Tras contestar a las preguntas sobre su perfil, debían valorar la audioguía. La mayoría de las preguntas eran cerradas, en una escala de diferencial semántico de 10 puntos. Se imitó el cuestionario QUIS, en el que los dos polos suelen ser calificados con etiquetas y se asigna a 0 el valor negativo y a 9, el positivo. Tras cada bloque (interfaz, guion y narración) se incluía una pregunta abierta para que los sujetos introdujeran sus sugerencias de una manera menos pautada. La Tabla 2 resume las puntuaciones obtenidas sobre la interfaz. 


\begin{tabular}{|c|c|c|c|c|c|}
\hline Tema & Foco & Cód. & Valor 0 & Valor 9 & Media \\
\hline & \multirow{5}{*}{ Descripción } & $2 a$ & Horrible & Estupenda & 8 \\
\hline & & $2 b$ & Frustrante & Satisfactoria & 8.25 \\
\hline & & $2 c$ & Aburrida & Estimulante & 7.25 \\
\hline & & $2 d$ & Rígida & Flexible & 7.5 \\
\hline & & $2 e$ & Incompleta & Completa & 8.25 \\
\hline \multirow{2}{*}{ Pantalla } & Legibilidad de los caracteres & $2 f$ & Poca & Mucha & 8 \\
\hline & Información & $2 g$ & Confusa & Diáfana & 8.5 \\
\hline \multirow{4}{*}{ Terminología } & General & $2 h$ & Inconsistente & Consistente & 7.5 \\
\hline & Encaje & $2 i$ & Nunca & Siempre & 8 \\
\hline & Posición de los mensajes & $2 j$ & Inconsistente & Consistente & 8.5 \\
\hline & Lenguaje & $2 k$ & Enrevesado & Claro & 8.5 \\
\hline \multirow{3}{*}{ Uso } & Acceso & $2 l$ & Difícil & Fácil & 8.5 \\
\hline & Aprendizaje & $2 m$ & Difícil & Fácil & 8.75 \\
\hline & Estructura & $2 n$ & Confusa & Lógica & 8.5 \\
\hline
\end{tabular}

Tabla 2. Valoraciones respecto a la interfaz

En general, todos los valores tienden al polo positivo. Sobre todo, encuentran que la audioguía es satisfactoria (2b) y completa (2e). Sobre la pantalla, consideran que los caracteres son legibles $(2 f)$ y que la organización de la información es clara (2g). Este bloque recogía asimismo dos preguntas dicotómicas de Sí/No:

- que todo figure en mayúsculas les pareció bien a tres de los sujetos, y

- los cuatro consideraron un acierto que la información esté dispuesta en una sola página, ya que ello permite una visión global de la audioguía.

De la terminología destacan el lenguaje claro (2k) y la consistencia en la posición de los mensajes (2)). Por último, les resulta fácil tanto acceder a ella (2l) como aprender a usarla $(2 \mathrm{~m})$. Según la información recogida mediante las preguntas abiertas, la interfaz debería hacer accesible el avance y retroceso del reproductor de los audios. Por lo demás, se incluyen felicitaciones por la incorporación de los tres idiomas, por el estilo conciso y claro de la interfaz y por haber conseguido reflejar la posición correcta de cada rincón del edificio.

La Tabla 3 muestra los resultados del segundo bloque, referido al guion. 


\begin{tabular}{|c|c|c|c|c|}
\hline Foco & Cód. & Valor 0 & Valor 9 & Media \\
\hline Contenido & $2 \tilde{n}$ & Incompleto & Completo & 8 \\
\hline Lugares básicos (entradas y salidas, baños, cafetería, etc.) & 20 & Nunca & Siempre & 8.5 \\
\hline Orientaciones sobre la iluminación & $2 p$ & Nunca & Siempre & 5.5 \\
\hline Ojustado a lo esperable para un espacio de este tipo & $2 q$ & Nunca & Siempre & 8.25 \\
\hline Orden lógico & $2 r$ & Nunca & Siempre & 8.5 \\
\hline $\begin{array}{c}\text { Organización sencilla y consistente } \\
\text { Lenguaje claro y sencillo (evita abreviaturas, jergas, } \\
\text { extranjerismos, verborrea y conceptos abstractos; define } \\
\text { términos especializados; usa la voz activa, palabras cortas, } \\
\text { ejemplos prácticos, adjetivos concretos, lenguaje directo, } \\
\text { respetuoso y positivo) }\end{array}$ & $2 t$ & Nunca & Siempre & 8.25 \\
\hline $\begin{array}{c}\text { Se limita a describir y evita las explicaciones } \\
\text { Léxico específico }\end{array}$ & $2 u$ & Nunca & Siempre & 7 \\
\hline
\end{tabular}

Tabla 3. Valoraciones respecto al guion

Los usuarios celebran que se incluyan instrucciones para llegar a lugares básicos (20), el orden (2r) y la organización de la información (2s), que les resulta completa (2n). Creen que el lenguaje del guion es claro y sencillo (2t). Señalan que apenas se incluyen orientaciones sobre la iluminación $(2 p)$ y han calificado con 7 el criterio $2 u$, tal vez porque algún informante no entendió la pregunta o porque dicha pregunta estaba mal formulada: quizás no quedaba claro que las dos afirmaciones unidas con la conjunción y se refieren a la misma característica de la audioguía (un guion en el que predomine la descripción pura sobre la explicación) o tal vez costaba discernir cuál de los dos polos (Nunca o Siempre) tenía una carga positiva para el investigador. En las preguntas abiertas de este bloque destacan de nuevo el lenguaje «fácilmente entendible». Algunas críticas individuales son:

- A considera que hay demasiada información respecto a los metros: sería preferible ir al grano y no marcar la distancia que hay en todos los espacios.

- B incluiría más información sobre la rugosidad del pavimento y también sobre las papeleras de reciclaje.

- D cree que se puede diferenciar mejor los pasillos derecho e izquierdo principales.

Por último, la Tabla 4 muestra los resultados del último bloque, sobre la narración. 


\begin{tabular}{|c|c|c|c|c|}
\hline Foco & Cód. & Valor 0 & Valor 9 & Media \\
\hline Voz & $2 w$ & Confusa & Clara & 8.75 \\
\hline Tono & $2 x$ & Afectado & Neutro & 9 \\
\hline Volumen & $2 y$ & Inadecuado & Adecuado & 9 \\
\hline Grabación & $2 z$ & Entrecortada & Fluida & 9 \\
\hline
\end{tabular}

Tabla 4. Valoraciones respecto a la narración

Este bloque es el mejor valorado, pues tres de los cuatro criterios incluidos obtienen la máxima puntuación posible y el otro, la segunda máxima puntuación. Las preguntas abiertas correspondientes abundan en esta buena percepción. Para B, la narración ha sido realizada de forma impecable en todos los idiomas y por todos los locutores. A considera que el ritmo de los audios podría ser algo más vivo; en cambio, D habla de un tono y un ritmo excelentes.

La información recabada a partir de las opiniones de los usuarios fue comprobada también con las herramientas utilizadas durante la última fase de la valoración, cuyos resultados se ofrecen en la $\S 4.3$.

\subsection{Eje económico}

Para terminar, la tercera comprobación mide el eje económico; esto es: si el resultado compensa el esfuerzo y los recursos dedicados. Para medir la experiencia de acuerdo con este eje, se consultó a los miembros del equipo de innovación, siguiendo la metodología empleada por Remael et al. (2019), en la que intervienen en la autoevaluación del proyecto todas las instituciones participantes.

Mendoza y Matamala (2019) describen el protocolo creado para medir la satisfacción de los profesores que pusieron en marcha su proyecto sobre AD. Dicho protocolo consistió en una encuesta anonimizada -compuesta mayoritariamente por preguntas abiertas-, realizada de manera telemática, grabada y transcrita después de que los participantes firmaran un consentimiento informado. Otros trabajos se basaron en grupos focales. En Agulló et al. (2018) se muestra una sesión con preguntas abiertas con las que se pretendía provocar un debate vivo; por su parte, Zhang (2019) entrevistó al equipo artístico, tanto de manera individual como en grupo, para pulsar opiniones sobre la accesibilidad de todos aquellos que habían tomado parte en la producción de teatro.

Los antecedentes aconsejaban, pues, una sesión en forma de grupo focal y contenidos orientados al proceso de creación de la audioguía. Por consiguiente, se programó una sesión de discusión virtual para el 17 de diciembre de 2020, a la que, a excepción de TP, asistieron todos los miembros del PIE: CO, la profesora de traducción (TR), la profesora de interpretación (IN), la becaria (BE) y las 3 profesoras de arte (A1, A2 y A3). En el mensaje en el que se les citaba se incluía un enlace a la audioguía terminada y se les pedía que la revisaran. Asimismo, se requería su consentimiento para grabar la parte de la sesión dedicada a la autoevaluación del proyecto. Obtenido dicho 
consentimiento, no hubo inconveniente para que, a mitad de la reunión (celebrada de forma remota a través de Blackboard Collaborate), se accionara la grabación que se transcribiría más tarde para analizar manualmente la información y redactar este resumen. La estructura del grupo focal tenía dos partes: lista de comprobación y preguntas abiertas.

\subsubsection{Lista de comprobación}

En primer lugar, se repasó una lista de cuestiones que habían quedado pendientes. La lista (Tabla 5), dividida en pasos — como los marcados en las guías del CESyA (2013, 2008)_, incluía una columna con la opinión previa del CO y otra con la del grupo (GR).

\begin{tabular}{|c|c|c|c|c|}
\hline Paso & Cód. & Criterio & $\mathrm{CO}$ & GR \\
\hline \multirow{3}{*}{ Análisis } & $3 a$ & Factible (Disponible) & $s$ & $\checkmark$ \\
\hline & $3 b$ & Factible (Abarcable) & 8 & $\checkmark$ \\
\hline & $3 c$ & Posibilidad de elegir el contenido & $x$ & $\checkmark$ \\
\hline \multirow{7}{*}{ Guion } & $3 d$ & Integridad & $s$ & $\checkmark$ \\
\hline & $3 e$ & Inmediatez & 8 & $\sqrt{2}$ \\
\hline & $3 f$ & Documentación disponible y facilitada a los descriptores & $x$ & $\checkmark$ \\
\hline & $3 g$ & Adecuación al usuario potencial & $\checkmark$ & $\checkmark$ \\
\hline & $3 h$ & Letreros & $x$ & $x$ \\
\hline & $3 i$ & Audiodescriptores familiarizados con la nomenclatura básica & $x$ & $\checkmark$ \\
\hline & $3 j$ & Lenguaje sencillo & $s$ & $\checkmark$ \\
\hline Revisión & $3 k$ & Realizada por personas diferentes a los redactores & $\checkmark$ & $\checkmark$ \\
\hline \multirow{4}{*}{ Narración } & $3 l$ & Voz clara & $s$ & $\checkmark$ \\
\hline & $3 m$ & Tono natural & $\checkmark$ & $\checkmark$ \\
\hline & $3 n$ & Locutor humano & $s$ & $\checkmark$ \\
\hline & $3 \tilde{n}$ & Cercanía del locutor a la obra & s & $\checkmark$ \\
\hline
\end{tabular}




\begin{tabular}{|c|c|c|c|c|}
\multirow{4}{*}{ Montaje } & 30 & Volumen fluido & $\checkmark$ & $\checkmark$ \\
\cline { 2 - 6 } & $3 p$ & Con introducción, anuncios entre cortes y vuelta al inicio & $\mathbf{x}$ & $\mathbf{x}$ \\
\cline { 2 - 6 } & $3 q$ & Facilidad de uso & $\checkmark$ & $\checkmark$ \\
\hline Evaluación & $3 r$ & Por personas con discapacidad visual & $\checkmark$ & $\checkmark$ \\
\hline
\end{tabular}

Tabla 5. Lista de comprobación del grupo focal

CO había premarcado positivamente todos los criterios salvo cuatro. Sobre la fase de Análisis, consideró que los responsables no pudieron elegir el contenido descriptible (3c), ya que este venía dado por la fisonomía del edificio. No obstante, el grupo señaló que sí se tomaron decisiones sobre qué espacios cubrir (por ejemplo, se decidió prescindir de la descripción de la cafetería).

Respecto al Guion, CO tenía dudas en los criterios $3 f$ (disponibilidad de la información), 3h (letreros) y 3i (familiarización con la nomenclatura). A1 corrigió las dos últimas cuestiones al recordar que los guionistas recibieron mapas de la facultad, así como un glosario para asegurar la uniformidad en el vocabulario de los textos. IN informó de que las personas ciegas que participaron en el inicio del proyecto preferían que no se cargaran las descripciones con la lectura de los letreros, los cuales, además, están escritos en Braille. Por esa razón, la exclusión de los letreros quedaba justificada.

En el paso de Montaje, CO no estaba seguro de que se hubieran incorporado partes introductorias, anuncios entre cortes y la posibilidad de volver al principio de cada grabación. IN confirmó que algunos locutores habían manifestado la opinión de que algunos cortes quedaban demasiado extensos: al comprobar este punto se vio que, efectivamente, cuatro cortes superan los cinco minutos que suelen recomendarse como máximo en la AD museística (Giansante, 2003). Por otra parte, el grupo admitió que no se planteó realizar introducción o explicación de la audioguía, la cual queda pendiente.

Aparte de las dudas, con esta primera parte del grupo focal se destacaron puntos fuertes de la audioguía, como el uso de locutores humanos -es más, nativos en los tres idiomas- en lugar de la impersonal voz sintética.

\subsubsection{Preguntas abiertas}

A continuación, CO moderó la discusión suscitada por una serie de preguntas abiertas. Como en toda esta fase, los participantes tenían libertad para intervenir (o no hacerlo) sin un orden fijado de antemano y como reacción al guion que CO había preparado en torno a los cuatro puntos recogidos en Zhang (2019): aprendizaje, concienciación, enfoque $y$ actitud.

Sobre el aprendizaje, todos están de acuerdo en que ha aumentado su conocimiento sobre las audioguías, sobre todo entre aquellos que carecían de experiencia en accesibilidad. 
Respecto al segundo punto, la mayoría considera que están ahora más concienciados, aunque la mejora percibida es menor que en el primer punto, ya que la conciencia sobre el problema es precisamente lo que hizo que algunos de ellos se acercaran al proyecto. Se destaca el beneficio para la institución; en palabras de A2: «es un ejemplo de que se puede aunar la actividad docente con la praxis profesional e incluso con la investigación». A1 y A3 reconocen que el proyecto ha servido para enriquecer sus clases: los nuevos contenidos son apreciados por los alumnos e incluso se han realizado varios trabajos de fin de grado sobre el tema. Asimismo, otros colectivos ajenos al proyecto pueden aumentar su concienciación para con la accesibilidad, sobre todo una vez que la página web está terminada y cabe difundirla: la audioguía es una invitación a adentrarse en el mundo de la accesibilidad para las personas ciegas.

Con la tercera cuestión se recogían opiniones sobre los puntos fuertes y débiles del proyecto. Como punto débil, A3 señala que algunos participantes partían de unos conocimientos tan escasos sobre la $A D$ que los alumnos que audiodescribieron el edificio se sintieron perdidos, lo que llevó a que los textos fueran parcialmente reescritos por las profesoras. La limitación temporal que marcaba la convocatoria del proyecto hizo además que las traducciones - que iban a correr a cargo de los alumnos del grado de traducción - tuvieran que ser realizadas por estudiantes de prácticas. Esta modificación no afectó en principio a la audioguía como producto, pero sí al potencial formativo de los alumnos del grado de traducción, que eran uno de los grupos que mayor interés podían tener en vivir la experiencia de innovación educativa. Otro percance derivado de la pandemia fue que, en los últimos estadios del proyecto, IN tuvo que gestionar la locución de manera telemática.

Las virtudes principales son el aprendizaje trasladado a las asignaturas y el hecho de que el producto, después de todos los problemas surgidos, haya visto la luz. A1 y TR destacan la integración en un mismo proyecto de técnicos, alumnos, becarios y profesores. TR destaca también que, aunque la dotación económica era limitada, la implicación humana fue enorme. Para $\mathrm{CO}$, por mucho que el proceso haya sido mejorable (sobre todo en su propio caso), el resultado es más que digno.

La última pregunta aludía a los beneficios para el futuro profesional del equipo. Para $\mathrm{CO}$ ha supuesto un descubrimiento y espera que el proyecto se convierta en una línea de investigación estable en su carrera. A2 está de acuerdo y considera que también se plantea seguir con la línea de investigación. A1 y A3, en cambio, creen que el proyecto afectará a su carrera docente, pero no a su labor investigadora. En cuanto a la continuidad, A1 se abstiene, dado que no imparte ya la asignatura que impartía cuando se inició el proyecto. El resto se compromete a buscar fórmulas que permitan mejorar la audioguía y el protocolo de creación para extrapolarlo, si cabe, a otros espacios.

\section{Conclusión}

La interacción persona-ordenador permite a los usuarios con discapacidad disfrutar de contenidos que antes les eran inaccesibles. Dada su relevancia social, la AD y otras modalidades de accesibilidad (como la subtitulación para personas sordas) están cada 
vez más presentes en los entornos formativos de la traducción, donde a menudo se las ha abordado como herramientas para la innovación educativa.

Este trabajo culmina un proyecto de innovación educativa cuyo objetivo era la creación de una audioguía accesible y multilingüe de la Facultad de Letras de la UPV/EHU. Tras definir los términos implicados, se ha descrito el proceso técnico de creación, el estado actual de la página donde se aloja y el protocolo seguido para evaluarla.

Los tres ejes de calidad medidos han arrojado resultados positivos. Desde un punto de vista normativo, la audioguía cumple con las recomendaciones existentes; cuando no lo hace, se ha preguntado a los usuarios, que aprueban las decisiones tomadas. La respuesta de dichos usuarios, que han incluido ideas para mejorar la audioguía, ha sido óptima. Finalmente, la puesta en común realizada por el equipo demuestra que la experiencia, en general, ha resultado fructífera.

Aparte de esto, el trabajo apunta hacia un modelo de evaluación de la calidad de la $A D$ en la que urgía profundizar (García-Prieto, 2020; Mazur y Vercauteren, 2019; Mendoza y Matamala, 2019), sobre todo en los entornos académicos. Este trabajo puede inspirar asimismo la puesta en marcha de audioguías similares en otros campos y espacios e incluso la creación de recursos y metodologías específicas para crear este tipo de textos.

No obstante, el proyecto tiene también limitaciones: desde la influencia de la pandemia, que ha complicado la ejecución de algunas fases del estudio, hasta el limitado número de usuarios que han probado la audioguía, pasando por las restricciones presupuestarias y temporales con las que ha tenido que trabajar un equipo que, por lo demás, ha tenido que ir aprendiendo sobre la materia mientras avanzaba.

Para futuras incursiones en el tema, fundamental -si bien difícil- será contar con usuarios nativos que prueben la audioguía en diferentes idiomas. A medio plazo, se deben corregir los defectos detectados y continuar implicando a personas e instituciones para que los proyectos sobre accesibilidad en general, y $A D$ en particular, sean vistos más como algo cotidiano que como producto de la innovación.

La tecnología es un apoyo clave tanto para la formación de traductores como para su desempeño profesional. Cuando la tecnología se pone al servicio de la accesibilidad, los beneficios son dobles, pues a las ventajas habituales hay que añadir las del compromiso ético.

\section{Referencias bibliográficas}

ADLAB PRO (2017). Assessment of current AD training practices. 〈http://www.adlabpro.eu/wpcontent/uploads/2017/12/20170608_uam_io1_report.pdf〉. [Accessed: 20210606].

AENOR (2005). Audiodescripción para personas con discapacidad visual. Requisitos para la audiodescripción y elaboración de audioguías. Madrid: AENOR. 
Agulló, Belén; Matamala, Anna; Orero, Pilar (2018). From disabilities to capabilities: testing subtitles in immersive environments with end users. Hikma, v. 17, pp. 195220. 〈https://www.uco.es/ucopress/ojs/index.php/hikma/article/view/11167〉, 〈https://doi.org/10.21071/hikma.v17i0.11167〉. [Accessed: 20210606].

Álvarez Álvarez, Ma de las Nieves (2014). Subtitulado: videosignado y audiodescripción en la España televisiva actual. Historia y Comunicación Social, v. 19, n. esp. 2, pp. 161-172. 〈https://revistas.ucm.es/index.php/HICS/article/view/45018/42389〉, $\langle$ https://doi.org/10.5209/rev_HICS.2014.v19.45018〉. [Accessed: 20210606].

Álvarez de Morales Mercado, Cristina (2017). Didáctica de la traducción accesible en el turismo y su aplicación en enseñanzas de posgrado. Revista Digital de Investigación en Docencia Universitaria, v. 11, n. 2, pp. 223-236. 〈http://www.scielo.org.pe/pdf/ridu/v11n2/a14v11n2.pdf〉, $\langle$ https://doi.org/10.19083/ridu.11.533〉. [Accessed: 20210606].

Cacheiro Quintas, Laura (2019). Diseño de un programa de subtitulación de vídeos pedagógicos [Tesis doctoral]. Universitat Jaume I. Castelló de la Plana. $\langle$ https://www.tdx.cat/handle/10803/668558\#page=1〉, 〈https://doi.org/10.6035/14110.2019.762203〉. [Accessed: 20210606〉.

Carlucci, Laura; Seibel, Claudia (2016). Universidad, accesibilidad y nuevas tecnologías: valoración de una experiencia de innovación docente en la traducción especializada. DIM: Didáctica, Innovación y Multimedia, n. 33, pp. 1-16. 〈https://www.raco.cat/index.php/DIM/article/view/306808〉. [Accessed: 20210606].

- (2014). El museo accesible: un nuevo espacio para el aprendizaje y la formación de estudiantes de Traducción. Trans-kom, v. 7, n. 1, p. 50-63. 〈http://www.transkom.eu/bd07nr01/trans-kom_07_01_03_Carlucci_Seibel_Museo.20140606.pdf〉. [Accessed: 20210606].

Castro Fernández, Daniel; Cosío Piccone, Gianella; Hu Huang, Diana; Calderón Díaz, Silvia (2018). Evaluación del proceso y producto de una propuesta de AD de tres obras de la exhibición permanente de arte moderno del museo de arte de Lima. Sendebar, v. 29, pp. 147-177.

〈https://revistaseug.ugr.es/index.php/sendebar/article/view/6745/7059〉, $\langle$ https://doi.org/10.30827/sendebar.v29i0.6745〉. [Accessed: 20210606].

Cerezo Merchán, Beatriz; De Higes Andino, Irene (2013). Trabajo colaborativo y desempeño profesional: un caso práctico en la clase de accesibilidad audiovisual. Hikma, v. 12, pp. 65-85. 〈https://www.uco.es/ucopress/ojs/index.php/hikma/article/view/5236/4917〉, $\langle$ https://doi.org/10.21071/hikma.v12i.5236〉. [Accessed: 20210606].

CESyA (2013). Guía de accesibilidad al teatro a través del subtitulado y la audiodescripción. Madrid: Real Patronato sobre Discapacidad: Ministerio de Sanidad, Servicios Sociales e Igualdad. 〈https://sid.usal.es/idocs/F8/FDO26587/accesibilidad_teatro.pdf〉. [Accessed: 20210606]. 
- (2008). Guías multimedia accesibles: el museo para todos. Madrid: Real Patronato sobre Discapacidad. 〈https://sid-

inico.usal.es/idocs/F8/FDO21399/museo_para_todos.pdf $\rangle$. [Accessed: 20210606].

Conde Ruano, José Tomás (en prensa). Calidad. Enciclopedia de Estudios de Traducción e Interpretación. Granada: AIETI.

Díez Carbajo, Coral (2018). Por una mejora de la usabilidad y utilidad en las herramientas de postedición. Clina, v. 4, n. 2, pp. 123-140. 〈https://revistas.usal.es/index.php/clina/article/view/clina201842123140/21025〉, $\langle$ https://doi.org/10.14201/clina201842123140〉. [Accessed: 20210606].

García-Prieto, Victoria (2020). La audiodescripción en televisión lineal y bajo demanda: el caso de TVE. TRANS, n. 24, pp. 129-144. 〈https://revistas.uma.es/index.php/trans/article/view/7484/11706〉, 〈https://doi.org/10.24310/TRANS.2020.v0i24.7484〉. [Accessed: 20210606].

Giansante, Lou (2003). Writing Verbal Descriptions for Audio Guides. Art Beyond Sight. 〈http://www.artbeyondsight.org/mei/wp-content/uploads/Writing-for-Audio-Guidesshort.pdf. [Accessed: 20210606].

Hermosa-Ramírez, Irene (2020). Delivery approaches in audio description for the scenic arts. Parallèles, v. 32, n. 2, pp. 17-31. <https://www.paralleles.unige.ch/files/9116/0311/9421/Paralleles-32-2_HermosaRamirez.pdf〉, 〈https://doi.org/10.17462/para.2020.02.02〉. [Accessed: 20210606].

López-Rodríguez, Clara Inés; Prieto-Velasco, Juan Antonio; Tercedor, Maribel (2009). Sharing environmental information through multimedia terminological and multimedia resources: the role of accessibility in increasing public awareness towards sustainable growth. In: Hřebíček, Jiří; Hradec, Jiří; Pelikán, Emil; Mírovský, Ondřej; Pillmann, Werner; Holoubek, Ivan; Bandholz, Thomas (eds.). Towards eEnvironment. Opportunities of SEIS and SISE: Integrating Environmental Knowledge in Europe. Prague: Masaryk University.

Luque Colmenero, M. Olalla; Soler Gallego, Silvia (2019). Training audio describers for art museums. Linguistica Antverpiensia, New Series: Themes in Translation Studies, n. 18, pp. 166-181. 〈https://lans-tts.uantwerpen.be/index.php/LANSTTS/article/view/509/451〉. [Accessed: 20210607].

Matamala, Anna; Orero, Pilar (2016). Accessible Culture and Training (ACT): Defining a new professional profile. (Unlimited! International Symposium on Accessible Live Events). Antwerp. 〈https://ddd.uab.cat/pub/presentacions/2016/150096/Unlimited16_ACT_final.pdf〉 [Accessed: 20210605].

- (2007). Designing a course on Audio Description: main competences of the future professional. Linguistica Antverpiensia, New Series: hemes in Translation Studies, n. 6, pp. 329-344. 〈https://lans-tts.uantwerpen.be/index.php/LANSTTS/article/view/195/126>. [Accessed: 20210605]. 
Mazur, Iwona; Vercauteren, Gert (2019). Media accessibility training. Linguistica Antverpiensia, New Series: hemes in Translation Studies, n. 18, pp. 1-22. 〈https://lans-tts.uantwerpen.be/index.php/LANS-TTS/article/view/564/464〉. [Accessed: 20210605].

Mendoza Domínguez, Nuria; Matamala, Anna (2019). Panorama de la enseñanza de la audiodescripción en España: Resultados de un cuestionario. MonTI, v. 11, pp. 155185. 〈https://www.e-revistes.uji.es/index.php/monti/article/view/4263/3454〉, $\langle$ https://doi.org/10.6035/MonTI.2019.11.6〉. [Accessed: 20210605].

O'Brien, Sharon (2012). Translation as a human-computer interaction. Translation Spaces, v. 1, n. 1, pp. 1-26. 〈https://core.ac.uk/download/pdf/192552515.pdf》, 〈https://doi.org/10.1075/ts.1.05obr〉. [Accessed: 20210607].

ONCE (2003). Accesibilidad para personas con ceguera y deficiencia visual. Madrid: ONCE. 〈https:/ $/$ www.diba.cat/c/document_library/get_file?uuid=1f52cb9c-5861-415d95f3-2d0c710d4dc4\&groupld=7294824〉. [Accessed: 20210607].

Prieto Velasco, Juan Antonio (2009). La accesibilidad como competencia del traductor: propuesta de actividades para el aprendizaje autónomo. Sendebar, v. 20, pp. 201230. 〈https://revistaseug.ugr.es/index.php/sendebar/article/view/397/429〉, 〈https://doi.org/10.30827/sendebar.v20i0.397〉. [Accessed: 20210607].

Remael, Aline; Orero; Pilar; Black, Sharon; Jankowska, Anna (2019). From translators to accessibility managers: How did we get there and how do we train them? MonTl, v. 11, pp. 131-154. 〈https://www.erevistes.uji.es/index.php/monti/article/view/4262/3453>, $\langle$ https://doi.org/10.6035/MonTI.2019.11.5〉. [Accessed: 20210607].

Risku, Hanna; Windhager, Florian (2013). Extended Translation: A Sociocognitive Research Agenda. Target, v. 25, n. 1, pp. 33-45. 〈https://www.jbeplatform.com/content/journals/10.1075/target.25.1.04ris $\rangle$, 〈https://doi.org/10.1075/target.25.1.04ris〉. [Accessed: 20210607].

Sanz-Moreno, Raquel (2020). La formación del audiodescriptor en España: situación actual y retos futuros. TRANS, n. 24, pp. 145-164. 〈https://revistas.uma.es/index.php/trans/article/view/7582/11707〉, 〈https://doi.org/10.24310/TRANS.2020.v0i24.7582〉. [Accessed: 20210607].

Snyder, Joel (2008). Audio description. The visual made verbal. In: Díaz-Cintas, Jorge (ed.) The Didactics of Audiovisual Translation. Amsterdam [etc.]: John Benjamins, pp. 191-198.

Soler Gallego, Silvia; Chica Núñez, Antonio (2014). Museos para todos: evaluación de una guía audiodescriptiva para personas con discapacidad visual en el museo de ciencias. Revista Española de Discapacidad, v. 2, n. 2, pp. 145-167. 〈https://www.cedd.net/redis/index.php/redis/article/view/94/pdf_19〉, $\langle$ https://doi.ort/10.5569/2340-5104.02.02.08〉. [Accessed: 20210607].

Soler Gallego, Silvia; Luque Colmenero, M. Olalla (2016). Elaboración de un sistema de audioguiado para la aplicación UGRQR. In: Álvarez de Morales, Cristina; Jiménez 
Hurtado, Catalina (eds.) Patrimonio cultural para todos. Investigación aplicada en traducción accesible. Granada: Ediciones Tragacanto. (Traducción accesible), pp. 5576.

Tapia, María Nieves (2010). La propuesta pedagógica del “Aprendizaje-Servicio”: una perspectiva latinoamericana. Tzhoecoen, revista científica, n. 5, pp. 23-43. 〈https://www.clayss.org.ar/archivos/TZHOECOEN-5.pdf〉. [Accessed: 20210607].

Zhang, Xiaochun (2019). Accessibility manager: Creating a profile of a new profession. Linguistica Antverpiensia, New Series: Themes in Translation Studies, n. 18, pp. 7386. 〈https://lans-tts.uantwerpen.be/index.php/LANS-TTS/article/view/510/450〉. [Accessed: 20210607]. 